\title{
Graphene hyperlens for terahertz radiation
}

\section{Andryieuski, Andrei; Lavrinenko, Andrei; Chigrin, Dmitry N.}

\section{Published in:}

Physical Review B Condensed Matter

Link to article, DOI:

10.1103/PhysRevB.86.121108

Publication date:

2012

Document Version

Publisher's PDF, also known as Version of record

Link back to DTU Orbit

Citation (APA):

Andryieuski, A., Lavrinenko, A., \& Chigrin, D. N. (2012). Graphene hyperlens for terahertz radiation. Physical Review B Condensed Matter, 86(12), [121108]. https://doi.org/10.1103/PhysRevB.86.121108

\section{General rights}

Copyright and moral rights for the publications made accessible in the public portal are retained by the authors and/or other copyright owners and it is a condition of accessing publications that users recognise and abide by the legal requirements associated with these rights.

- Users may download and print one copy of any publication from the public portal for the purpose of private study or research.

- You may not further distribute the material or use it for any profit-making activity or commercial gain

- You may freely distribute the URL identifying the publication in the public portal

If you believe that this document breaches copyright please contact us providing details, and we will remove access to the work immediately and investigate your claim 


\title{
Graphene hyperlens for terahertz radiation
}

\author{
Andrei Andryieuski* and Andrei V. Lavrinenko \\ Department of Photonics Engineering, Technical University of Denmark, DK-2800 Kongens Lyngby, Denmark \\ Dmitry N. Chigrin \\ Institute of High-Frequency and Communication Technology, University of Wuppertal, Rainer-Gruenter-Strasse 21 FE, \\ D-42119 Wuppertal, Germany
}

(Received 13 July 2012; published 27 September 2012)

\begin{abstract}
We propose a graphene hyperlens for the terahertz $(\mathrm{THz})$ range. We employ and numerically examine a structured graphene-dielectric multilayered stack that is an analog of a metallic wire medium. As an example of the graphene hyperlens in action, we demonstrate an imaging of two point sources separated by a distance $\lambda_{0} / 5$. An advantage of such a hyperlens as compared to a metallic one is the tunability of its properties by changing the chemical potential of graphene. We also propose a method to retrieve the hyperbolic dispersion, check the effective medium approximation, and retrieve the effective permittivity tensor.
\end{abstract}

DOI: 10.1103/PhysRevB.86.121108

PACS number(s): 78.67.Pt, 78.67.Wj, 81.05.Xj

Rapidly developing terahertz (THz) science and technology has acquired a great deal of attention in recent years due to its enormous potential for spectroscopy, communication, defense, and biomedical imaging. ${ }^{1-3}$ The natural diffraction limit, however, restricts the resolution of the standard $\mathrm{THz}$ imaging systems to about a wavelength, which is relatively large $(300 \mu \mathrm{m}$ in the free space at $1 \mathrm{THz})$. To overcome this restriction one can use scanning near-field $\mathrm{THz}$ microscopy that allows for even submicrometer resolution in the scattering (apertureless) configuration, ${ }^{4}$ but such a technique is slow. Another solution is to use a metamaterial lens with artificially engineered properties, for example, a negative index lens ${ }^{5}$ or a hyperbolic-dispersion lens (hyperlens). ${ }^{6}$ While the negativeindex material lens is far from being implemented into imaging systems due to high losses and a narrow resonant frequency range, the hyperlens has been experimentally demonstrated in the microwave ${ }^{7}$ and optical ${ }^{8}$ regimes. The hyperlens is able to convert evanescent waves into propagating ones and to magnify a subwavelength image so that it can be captured by a standard imaging system, such as a microscope, for example.

A hyperlens usually consists of metal-dielectric layers (in ultraviolet and optical ranges) or of metallic wires (infrared and microwave ranges). Due to the employment of metal, the properties of the hyperlens cannot be tuned after fabrication. In contrast to metal, graphene, a two-dimensional material with striking electronic, mechanical, and optical properties, ${ }^{9}$ supports surface plasmon polaritons in the $\mathrm{THz}$ range $\mathrm{e}^{10,11}$ that are widely tunable by a change of graphene's electrochemical potential via chemical doping, or magnetic field or electrostatic gating. ${ }^{12}$ Many plasmonic effects and photonic applications of graphene have been proposed. ${ }^{13-18}$ Nevertheless, to the best of our knowledge, no graphene based hyperlens for the $\mathrm{THz}$ range has been proposed so far (however, a graphene and boron nitride hyperlens for the ultraviolet has been reported recently ${ }^{19}$ ).

In this Rapid Communication we propose to use structured graphene for the creation of a hyperlens in the $\mathrm{THz}$ range. To support our proposal we investigate the effective properties of the hyperbolic graphene wire medium and then construct a hyperlens out of it. We check numerically the performance of a full-size three-dimensional (3D) and its homogenized two-dimensional (2D) analog and demonstrate that it has the desired subwavelength resolution and magnification.

Several requirements have to be satisfied for constructing the hyperlens. ${ }^{20,21}$ First of all, an indefinite material (the permittivity tensor components have opposite signs) with strong cylindrical anisotropy should be used. Namely, the radial permittivity $\varepsilon_{r}$ should be negative $\left(\varepsilon_{r}<0\right)$ while the azimuthal permittivity $\varepsilon_{\theta}$ should be positive $\left(\varepsilon_{\theta}>0\right)$. In this case the in-plane isofrequency contour is hyperbolic:

$$
\frac{q^{2}}{\varepsilon_{\theta}}+\frac{\kappa^{2}}{\varepsilon_{r}}=1,
$$

where $q=k_{r} / k_{0}$ is the normalized radial wave-vector component, $\kappa=k_{\theta} / k_{0}$ is the normalized azimuthal wave vector, and $k_{0}=2 \pi / \lambda_{0}=\omega / c$ is the wave number in vacuum. Then the waves with $\kappa>1$, which are evanescent in vacuum, can propagate in the hyperbolic medium. Mathematically this means that for every $\kappa$ there exists a real-valued $q$. Moreover, the dependence $q(\kappa)$ should be as flat as possible. That ensures the same phase velocities for all spatial components (various $\kappa)$. There are two possibilities for satisfying this requirement: to select a material with either a large negative $\varepsilon_{r}$ or a small positive $\varepsilon_{\theta}$.

For high transmission propagation losses characterized by $[\operatorname{Im}(q)]$ should be as small as possible. For the waves with $\kappa \ll 1$ the radial wave vector reduces to $q \approx \sqrt{\varepsilon_{\theta}}$, so it is primarily $\varepsilon_{\theta}$ that determines losses. The incoupling and outcoupling of the waves to the ambient medium should also be efficient. For normally incident waves going from a dielectric with a refractive index $n$ onto a flat interface with a hyperbolic medium, the reflection coefficient is $R=\frac{n-q}{n+q}=\frac{n-\sqrt{\varepsilon_{\theta}}}{n+\sqrt{\varepsilon_{\theta}}}$, so in order to minimize reflection one has to match the azimuthal permittivity $\varepsilon_{\theta}$ with the permittivity $\varepsilon=n^{2}$ of the surrounding medium. This requirement limits the range of $\varepsilon_{\theta}$. In addition, to maximize the hyperlens transmission, the Fabry-Pérot resonance condition should be satisfied,

$$
R_{2}-R_{1}=\frac{m \lambda_{\mathrm{eff}}}{2}=\frac{\pi m}{q k_{0}},
$$


(a)

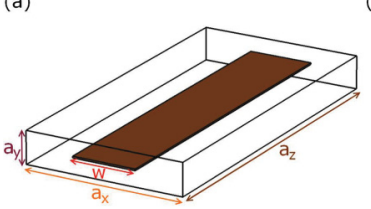

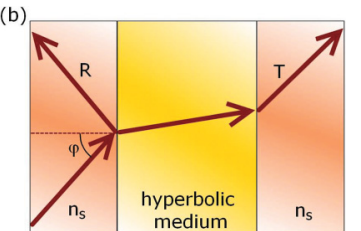

FIG. 1. (Color online) (a) The unit cell of the graphene wire medium consists of a graphene stripe of width $w$ embedded into a dielectric. (b) To characterize the hyperbolic medium we calculated the complex reflection $R$ and transmission $T$ coefficients for various angles of incidence $\varphi$. A block of the hyperbolic medium is placed between high-index $n_{s}$ dielectric layers.

where $R_{1}$ and $R_{2}$ are the inner and outer hyperlens radii, respectively, $\lambda_{\text {eff }}$ is the effective wavelength, and $m$ is an integer number. The ratio of the radii $M=R_{2} / R_{1}$ determines the hyperlens magnification.

Finally, since no natural electromagnetic materials with a strong cylindrical anisotropy exist, artificial effectively homogenous metamaterials have to be used. That means that its lateral geometrical period $P$ should be much (at least five to ten times) smaller than the period of the wave with the highest $\kappa=\kappa_{\max }$. So, for example, if we wish to construct the hyperlens for the free-space wavelength $\lambda_{0}=50 \mu \mathrm{m}$ that supports the wave with the highest $\kappa_{\max }=5$, then the lateral period of the metamaterial should not be larger than $P_{\max }=\frac{1}{10} \frac{\lambda_{0}}{\kappa_{\max }}=1 \mu \mathrm{m}$.

First we analyzed the properties of the graphene wire medium itself. Its unit cell is a rectangular block of a dielectric ( $\varepsilon_{D}=2.34$ corresponding to the low-loss polymer TOPAS) of the size $a_{x} \times a_{y} \times a_{z}=0.2 \times 0.05 \times 1 \mu \mathrm{m}^{3}\left(a_{x}, a_{y} \ll P_{\max }\right)$ with an embedded graphene stripe of width $w$ depicted in Fig. 1(a). We described graphene for the simulations in $\mathrm{CST}^{22}$ as a layer of thickness $\Delta=1 \mathrm{~nm}$ with the permittivity $\varepsilon_{G}=\varepsilon_{D}+i \frac{\sigma_{S}}{\varepsilon_{0} \omega \Delta}$, where $\sigma_{S}$ is the surface conductivity of graphene. $^{23}$

In order to retrieve the dispersion relation $q(\kappa)$ we simulated the complex reflection $R$ and transmission $T$ coefficients for various angles of incidence $\varphi$ on a hyperbolic medium slab [see Fig. 1(b)] with the periodic (unit cell) boundary conditions. We considered transverse magnetic (TM) polarized waves (magnetic field along the $y$ axis). The surrounding medium was a high refractive index $n_{S}$ dielectric. Then for each $\kappa$ and frequency $\omega$ we can restore $q,{ }^{24}$

$$
q= \pm \frac{1}{k_{0} a_{z}} \arccos \frac{1-R^{2}+T^{2}}{2 T}+\frac{2 \pi m}{k_{0} a_{z}},
$$

where $m$ is an integer number. Since we work in the long wavelength limit, the challenging choice of the branch $m$ is not an issue, and it should be simply $m=0$. The choice of the sign should satisfy the passivity condition $\operatorname{Im}(q)>0$. Knowing the dispersion dependence $q(\omega, \kappa)$, we can restore the components of the permittivity tensor $\varepsilon_{r}$ and $\varepsilon_{\theta}$ through the linear regression analysis of the dispersion equation (1),

$$
q^{2}=\varepsilon_{\theta}-\frac{\varepsilon_{\theta}}{\varepsilon_{r}} \kappa^{2} .
$$

The statistical coefficient of determination $R_{\mathrm{sq}}$ confirms (if $R_{\mathrm{sq}}$ close to 1$)$ the linear regression $q^{2}\left(\kappa^{2}\right)$ and the homogenous
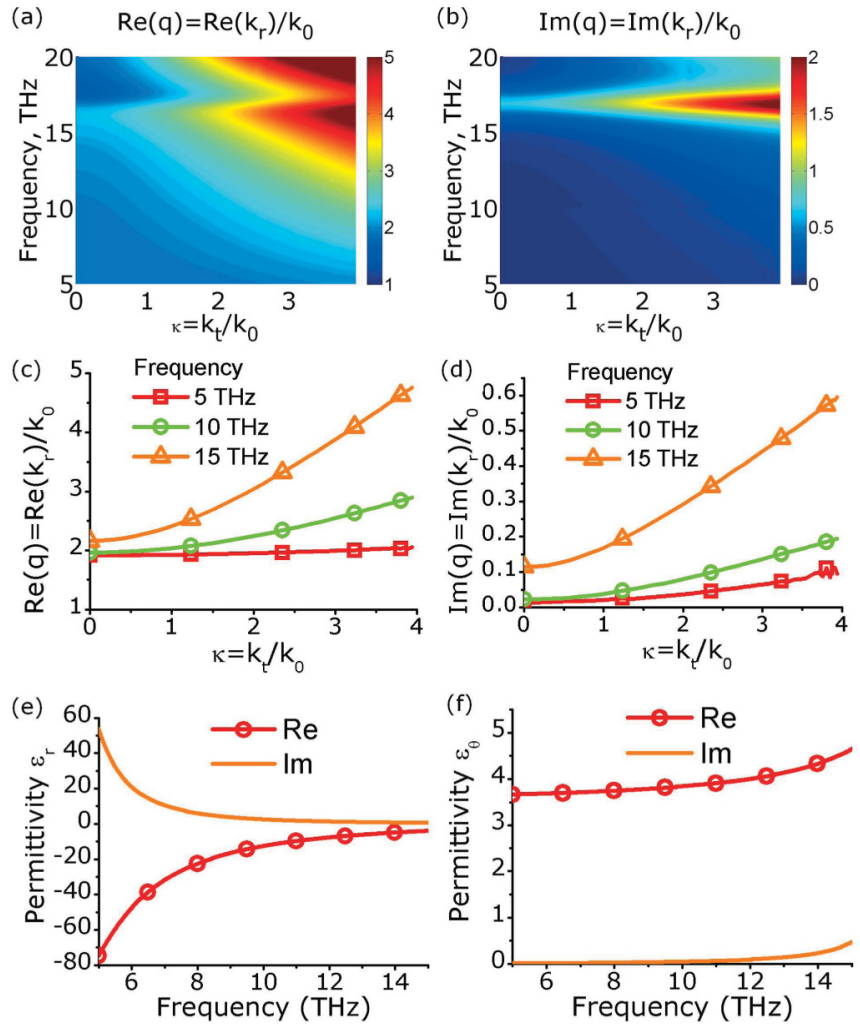

FIG. 2. (Color online) Contour plots of restored (a) $\operatorname{Re}[q(\omega, \kappa)]$ and (b) $\operatorname{Im}[q(\omega, \kappa)]$ for a graphene stripe of $w=80 \mathrm{~nm}$ show the absence of resonances at low frequencies, but a resonance at $f=$ $17 \mathrm{THz}$. Looking at the $q(\kappa)$ for certain frequencies $(5,10$, and $15 \mathrm{THz}$ ) we observe a flat $\operatorname{Re}[q(\kappa)]$ dependence (c) and smaller losses (d) for the frequency $5 \mathrm{THz}$. Effective radial permittivity $\varepsilon_{r}(\mathrm{e})$ shows Drude-like behavior, while azimuthal permittivity $\varepsilon_{\theta}$ (f) is positive with small losses.

approximation validity. For the investigated graphene wire medium we observed $R_{\text {sq }}>0.95$. We should also note that this retrieval method is applicable not only to the hyperbolic medium, but to any metamaterial, and that by selecting another polarization and/or wave propagation direction it is possible to restore the whole permittivity tensor.

An example of the restoration for the graphene stripe of width $w=80 \mathrm{~nm}$ is shown in Fig. 2. The color contour graphs [Figs. 2(a) and 2(b)] show that $q(\omega, \kappa)$ is flat at low frequencies, but exhibits a resonance around $17 \mathrm{THz}$. A detailed investigation of the electromagnetic field behavior revealed a surface plasmon resonance of the graphene stripe at this frequency. The $q(\kappa)$ isofrequency contours [Figs. 2(c) and 2(c)] are flatter and the losses are smaller at lower frequencies. Finally, the radial permittivity $\varepsilon_{r}$ has a Drude-like dependence [Fig. 2(e)] with large negative values at the low frequencies, while azimuthal $\varepsilon_{\theta}$ is positive and has $\operatorname{small} \operatorname{Im}\left(\varepsilon_{\theta}\right)$ [Fig. 2(f)]. Thus it is advantageous to select a low operation frequency for the hyperlens.

In order to select the optimal geometrical design, we investigated the dependence of the wire medium properties on the stripe width (see Fig. 3) starting from no graphene $(w=0)$ to a full graphene coverage $(w=200 \mathrm{~nm})$. As expected, in the absence of graphene we restore a constant refractive 

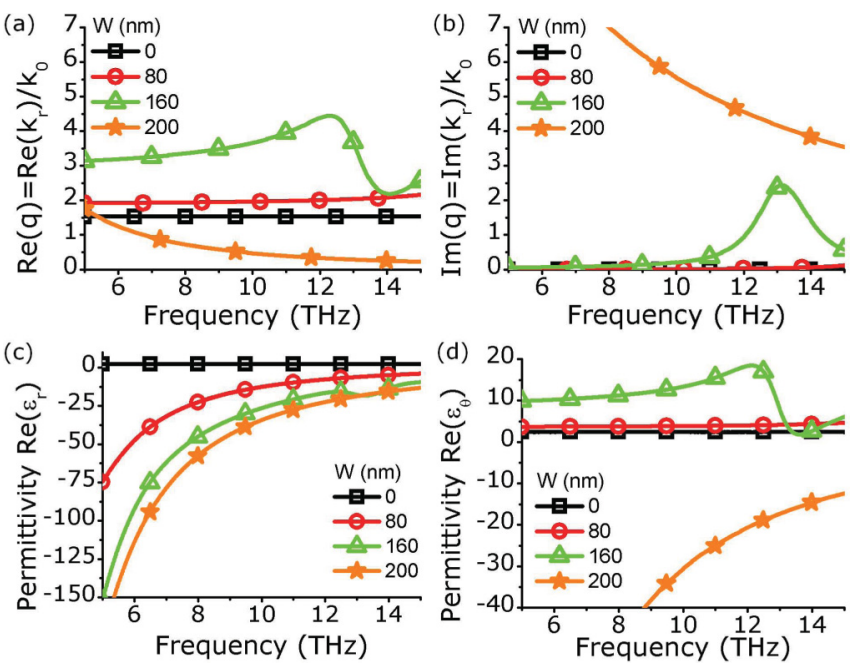

FIG. 3. (Color online) Comparison of the properties of the graphene wire medium for various stripe widths $(0,80,160$, and $200 \mathrm{~nm}$ ). The radial wave vector shows larger values of (a) $\operatorname{Re}(q)$ (that means worse coupling to the surrounding medium) and (b) $\operatorname{Im}(q)$ (larger losses) for $w=160 \mathrm{~nm}$ compared to the width $w=$ $80 \mathrm{~nm}$. Also, a "more metallic" Drude behavior of $\varepsilon_{r}$ (c) and higher azimuthal permittivity $\varepsilon_{\theta}$ (d) is observed for $w=160 \mathrm{~nm}$. The absence of graphene $(w=0)$ and full coverage $(w=200 \mathrm{~nm})$ show fully dielectric and Drude-like behaviors, respectively.

index $n_{D}=1.53$ [Fig. 3(a)] with no losses [Fig. 3(b)] and permittivities $\varepsilon_{r}=\varepsilon_{\theta}=n_{D}^{2}$ [Figs. 3(c) and 3(d)], while for full graphene coverage a typical Drude metal-like behavior is observed for permittivities $\varepsilon_{r}=\varepsilon_{\theta}$. Changing the width from $w=80 \mathrm{~nm}$, which we discussed above, to $w=160 \mathrm{~nm}$, we observe larger values of $\operatorname{Re}(q)$ for the normal propagation $\kappa=0$ [see Fig. 3(a)] (and consequently worse coupling efficiency), larger losses and redshift of the resonance to $f=13 \mathrm{THz}$ [Fig. 3(b)], and a larger negative permittivity $\varepsilon_{r}$ [Fig. 3(c)]. After examining several widths we selected for the hyperlens demonstration the width $w=40 \mathrm{~nm}$ (not shown in Fig. 3) and the frequency $6 \mathrm{THz}$.

To check the suitability of the effective medium approach we simulated in the CST (time domain) a full-size 3D hyperlens made of graphene stripes embedded into a dielectric $\left(n_{D}=\right.$ 1.53). One layer of the hyperlens is shown in Fig. 4(a). The input and output periods, widths, and radii were chosen as $P_{\text {in }}=200 \mathrm{~nm}, P_{\text {out }}=600 \mathrm{~nm}, W_{\text {in }}=40 \mathrm{~nm}, W_{\text {out }}=120 \mathrm{~nm}$, $R_{\text {in }}=15.12 \mu \mathrm{m}$, and $R_{\text {out }}=45.36 \mu \mathrm{m}$, respectively. The radii are selected to satisfy the Fabry-Pérot resonant condition (2). The layers of structured graphene are assumed to be periodic in the direction perpendicular to the image plane (period $a_{y}=50 \mathrm{~nm}$ ). We should note that the specified sizes are realistic for fabrication. Multiple graphene layers separated with a dielectric can be made up to a size of $30 \mathrm{in} .^{25}$ Structuring of a multiple graphene-dielectric layer structure can be done with focused ion beam milling or electron beam lithography.

Now we show the hyperlens in action when being excited by two sources (line magnetic currents) in vacuum separated with a distance $\delta=\lambda_{0} / 5=10 \mu \mathrm{m}$ [see the artistic 3D view of the hyperlens in work in Fig. 4(b)]. In the presence of the hyperlens the two sources are well resolved at the output interface as two peaks separated by $30 \mu \mathrm{m}$ [Fig. 4(c)]
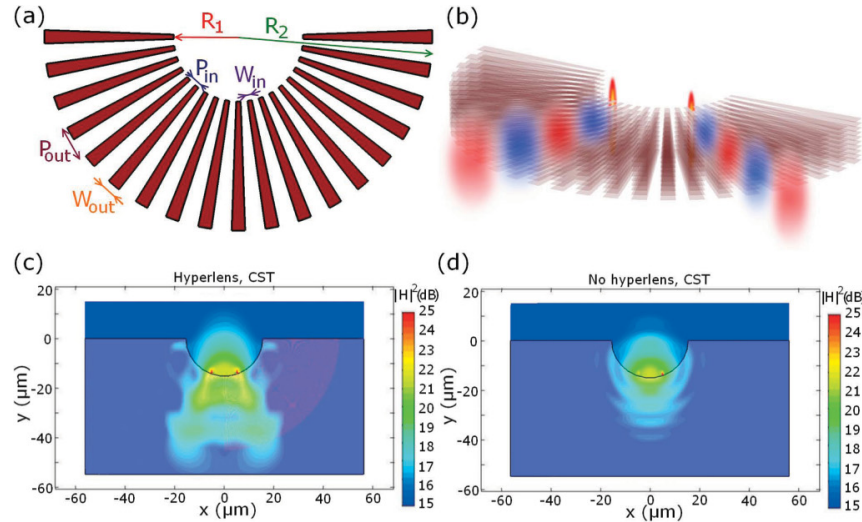

(d)
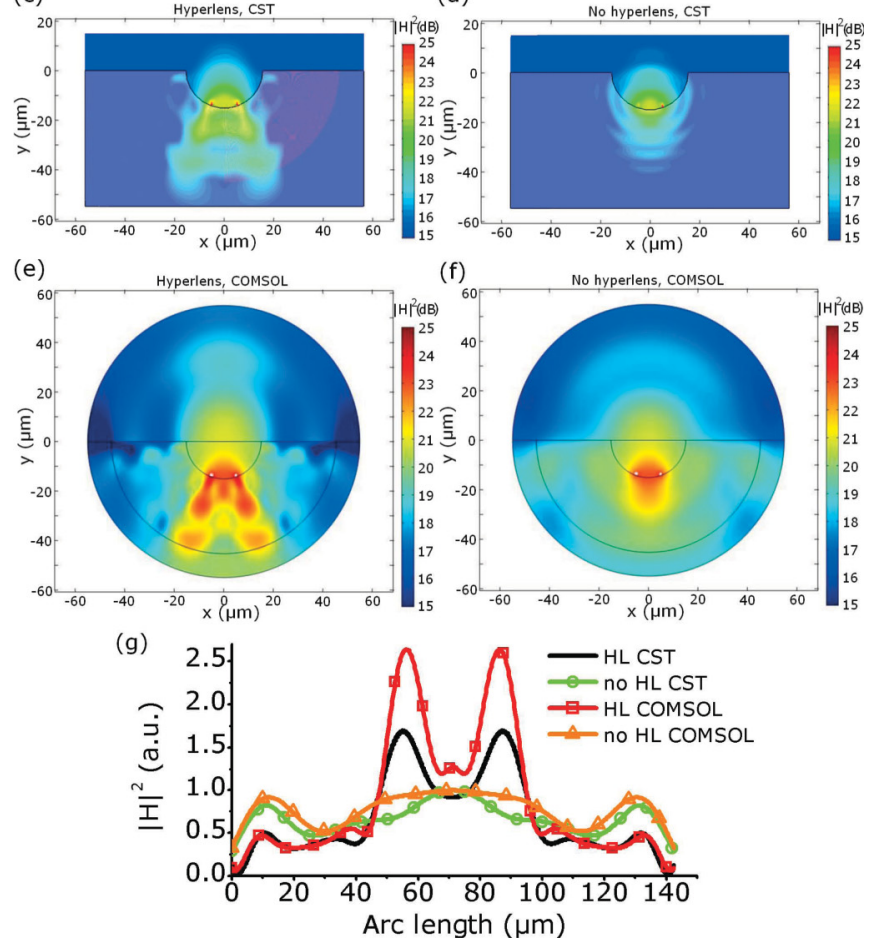

FIG. 4. (Color online) (a) A single structured graphene layer that constitutes the hyperlens. (b) An artistic 3D view of the hyperlens in action: The image of two line sources is magnified with the hyperlens. Full-size 3D CST simulation of the hyperlens (c) in action and no hyperlens (d), when two magnetic line sources separated by $\lambda_{0} / 5=10 \mu \mathrm{m}$ are emitting TM polarized waves (magnetic field perpendicular to the plane of image). The CST results are in a good agreement with equivalent 2D COMSOL simulations of the hyperlens (e) and no hyperlens (f). Comparison of the intensity distribution at the output interface of the hyperlens $(\mathrm{g})$ confirms that the images are well resolved.

delivering the magnification $M=R_{2} / R_{1}=3{ }^{26}$ while in the case of the homogenous dielectric cylinder (no graphene wires) we observe a single spot [Fig. 4(d)].

Then we compared the CST results with an equivalent 2D hyperlens simulation in $\mathrm{COMSOL}^{27}$ (scattering boundary conditions) with homogenized permittivities $\varepsilon_{r}=-20.1+8.5 i$, $\varepsilon_{\theta}=2.73+0.0029 i$. The COMSOL results with [Fig. 4(e)] and without the hyperlens [Fig. 4(f)] are in a good agreement with the CST results. A comparison between them is shown in Fig. 4(g), where the wave intensity at the output interface of the lens is presented. The intensity of the peaks in the presence of the hyperlens is larger than in its absence, due to a redistribution of the power. The intensity simulated with the CST is smaller compared to COMSOL that is caused by the coarser spatial discretization of the tapered wires with a staircase numerical mesh in the CST. In both types of simulations the peaks are well resolved according to the 

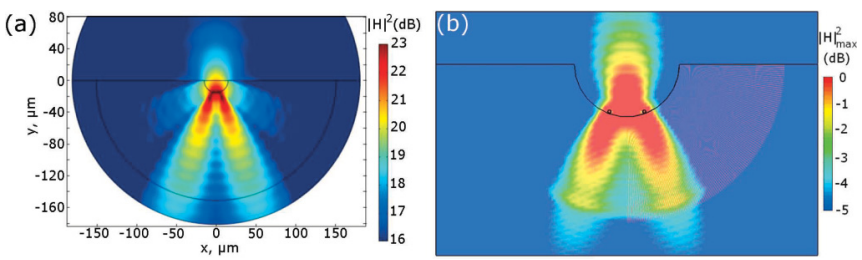

FIG. 5. (Color online) (a) A thicker hyperlens with $R_{2}=10 R_{1}$ magnifies two subwavelength sources until the images can be captured with a conventional $\mathrm{THz}$ imaging system. (b) Tracing the maximum of the broadband $\mathrm{THz}$ transient pulse shows that the hyperlens works in the range of frequencies around $6 \mathrm{THz}$.

Rayleigh criterion. The 2D COMSOL simulation, however, took several minutes versus the 3-days-long 3D CST modeling.

By making a hyperlens with a larger radius $R_{2}$, one can achieve a larger magnification. For example, selecting $R_{2}=10 R_{1}$ gives the magnification $M=10$, so two point sources with a separation $\delta=10 \mu \mathrm{m}$ are imaged to $100 \mu \mathrm{m}$ [see Fig. 5(a)] and then can be resolved with a conventional $\mathrm{THz}$ camera.

It is important to test the device performance under pulse excitation. In the conventional $\mathrm{THz}$ time domain spectroscopy setup $^{2}$ (THz-TDS), a very short (single cycle or even shorter) $\mathrm{THz}$ pulse is generated. Experimentally testing the hyperlens in the real THz-TDS would mean shining the short (and therefore broadband in frequency) transient pulse and then scanning with the $\mathrm{THz}$ near-field microscope and collecting the time-dependent signal at the output. We did a similar simulation in CST, exciting two sources with the Gaussian pulse [central frequency $f_{c}=6 \mathrm{THz}$, full width at half maximum $(\mathrm{FWHM})=12 \mathrm{THz}$, recording the field with the time monitor, and then imaging the maximal field in each point during the simulation time [Fig. 5(b)]. Since the graphene hyperlens is not based on a resonant medium, it can operate in an extended range of frequencies and two sources are well magnified and resolved [Fig. 5(b)].

Due to reciprocity, the hyperlens can be used not only for imaging, but also for $\mathrm{THz}$ power concentration into a small volume. We wish to note that the employment of metal for the considered hyperlens design is hardly possible. In order to obtain the same conductivity of the unit cell as of the regarded graphene stripes, the cross section of the metallic wire (for example, silver ${ }^{28}$ ) has to be of $2 \mu \mathrm{m}^{2}$. Fabricating and arranging such thin and long metallic wires into the required pattern is beyond the possibilities of current nanofabrication technologies. We should emphasize that scaling up the metallic wires together with the unit cell is not possible, since the period of the hyperlens should be subwavelength even for the higher-order spatial harmonics. Another important advantage of the graphene hyperlens compared to the metal based one is its tunability by the graphene chemical potential change. Thus it is possible to make the device reconfigurable and to resolve subwavelength features or concentrate $\mathrm{THz}$ pulses on demand.

In conclusion, we have shown that structured graphene layers embedded into a dielectric (graphene wire medium) can be used to create a hyperlens. We have proposed a realistic geometrical design for the hyperlens for the $\mathrm{THz}$ radiation and proved that it can resolve two line sources separated by a distance $\lambda_{0} / 5$. We also showed that time-consuming 3D simulations are in a good agreement with the quick homogenized 2D hyperlens modeling, which simplifies the hyperlens engineering.

The authors acknowledge A. Novitsky for useful discussions and M. Wubs for proofreading. A.A. acknowledges financial support from the Danish Council for Technical and Production Sciences through the GraTer (11-116991) project.

*andra@fotonik.dtu.dk

${ }^{1}$ M. Tonouchi, Nat. Photonics 1, 97 (2007).

${ }^{2}$ P. Jepsen, D. Cooke, and M. Koch, Laser Photonics Rev. 5, 124 (2011).

${ }^{3}$ T. Kleine-Ostmann and T. Nagatsuma, J. Infrared, Millimeter, Terahertz Waves 32, 143 (2011).

${ }^{4}$ R. Kersting, H.-T. Chen, N. Karpowicz, and G. C. Cho, J. Opt. A: Pure Appl. Opt. 7, S184 (2005).

${ }^{5}$ J. B. Pendry, Phys. Rev. Lett. 85, 3966 (2000).

${ }^{6}$ Z. Jacob, L. V. Alekseyev, and E. Narimanov, Opt. Express 14, 8247 (2006).

${ }^{7}$ P. A. Belov, Y. Hao, and S. Sudhakaran, Phys. Rev. B 73, 033108 (2006).

${ }^{8}$ Z. Liu, H. Lee, Y. Xiong, C. Sun, and X. Zhang, Science 315, 1686 (2007).

${ }^{9}$ N. Savage, Nature (London) 483, S30 (2012).

${ }^{10}$ M. Jablan, H. Buljan, and M. Soljačić, Phys. Rev. B 80, 245435 (2009).

${ }^{11}$ L. Ju, B. Geng, J. Horng, C. Girit, M. Martin, Z. Hao, H. A. Bechtel, X. Liang, A. Zettl, Y. R. Shen, and F. Wang, Nat. Nanotechnol. 6, $6(2011)$

${ }^{12}$ J. Chen, M. Badioli, P. Alonso-González, S. Thongrattanasiri, F. Huth, J. Osmond, M. Spasenović, A. Centeno, A. Pesquera, P. Godignon, A. Z. Elorza, N. Camara, F. J. García de Abajo, R. Hillenbrand, and F. H. L. Koppens, Nature (London) 487, 77 (2012).

${ }^{13}$ S. H. Lee, M. Choi, T.-T. Kim, S. Lee, M. Liu, X. Yin, H. K. Choi, S. S. Lee, C.-G. Choi, S.-Y. Choi, X. Zhang, and B. Min, arXiv:1203.0743 [Nature Mater. (to be published)].

${ }^{14}$ M. Liu, X. Yin, E. Ulin-Avila, B. Geng, T. Zentgraf, L. Ju, F. Wang, and X. Zhang, Nature (London) 474, 64 (2011).

${ }^{15}$ M. Liu, X. Yin, and X. Zhang, Nano Lett. 12, 1482 (2012).

${ }^{16}$ A. Vakil and N. Engheta, Science 332, 1291 (2011).

${ }^{17}$ P. Tassin, T. Koschny, M. Kafesaki, and C. M. Soukoulis, Nat. Photonics 6, 259 (2012).

${ }^{18}$ L. Ju, B. Geng, J. Horng, C. Girit, M. Martin, Z. Hao, H. A. Bechtel, X. Liang, A. Zettl, Y. R. Shen, and F. Wang, Nat. Nanotechnol. 6, 6 (2011).

${ }^{19}$ J. Wang, Y. Xu, H. Chen, and B. Zhang, J. Mater. Chem. 22, 15863 (2012).

${ }^{20}$ Z. Jacob, L. V. Alekseyev, and E. Narimanov, J. Opt. Soc. Am. A 24, A52 (2007). 
${ }^{21}$ M. G. Silveirinha, P. A. Belov, and C. R. Simovski, Phys. Rev. B 75, 035108 (2007).

${ }^{22}$ CST Computer Simulation Technology AG, http://cst.com.

${ }^{23}$ The surface conductivity of graphene was calculated with the Kubo formula (Ref. 29) in the random-phase approximation with the value of $\tau=10^{-13} \mathrm{~s}$ [which corresponds to a rather conservative value of mobility $\left.\mu=10^{4} \mathrm{~cm}^{2} /(\mathrm{V} \mathrm{s})\right]$, the temperature $T=300 \mathrm{~K}$, and Fermi level $E_{F}=0.5 \mathrm{eV}$. We compared the conductivity values that we used with the experimentally measured in the $\mathrm{THz}$ range (Ref. 30) and the relative difference was less than $7 \%$. Our test calculations for plasmon dispersion on a suspended graphene showed that numerical results differ from the analytical ones (Ref. 31) by less than $5 \%$ for the selected effective thickness $\Delta=1 \mathrm{~nm}$.

${ }^{24}$ C. Menzel, C. Rockstuhl, T. Paul, F. Lederer, and T. Pertsch, Phys. Rev. B 77, 195328 (2008).
${ }^{25}$ S. Bae, H. Kim, Y. Lee, X. Xu, J.-S. Park, Y. Zheng, J. Balakrishnan, T. Lei, H. R. Kim, Y. I. Song, Y.-J. Kim, K. S. Kim, B. Ozyilmaz, J.-H. Ahn, B. H. Hong, and S. Iijima, Nat. Nanotechn. 5, 574 (2010).

${ }^{26}$ We were limited by the computational power, so we took the hyperlens with a small magnification $M=R_{2} / R_{1}=3$ (still, a 12-core CPU with $48 \mathrm{~Gb}$ RAM executed the task in 3 days).

${ }^{27}$ COMSOL Inc., http://www.comsol.com/.

${ }^{28}$ N. Laman and D. Grischkowsky, Appl. Phys. Lett. 93, 051105 (2008).

${ }^{29}$ G. Hanson, IEEE Trans. Antennas Propag. 56, 747 (2008).

${ }^{30}$ L. Ren, Q. Zhang, J. Yao, Z. Sun, R. Kaneko, Z. Yan, S. Nanot, Z. Jin, I. Kawayama, M. Tonouchi, J. M. Tour, and J. Kono, Nano Lett. 12, 3711 (2012).

${ }^{31}$ L. A. Falkovsky, J. Phys.: Conf. Ser. 129, 012004 (2008). 